\title{
Circulatory collapse in a 30-year-old amenorrheic woman
}

\author{
R Goswami, N Tandon, B Singh, P Shah, AC Ammini
}

A 30-year-old woman presented to the emergency services of the hospital with drowsiness, poor appetite, constipation, postural giddiness and amenorrhea since the birth of her last child four years ago which was associated with post-partum haemorrhage. Examination revealed a blood pressure of $70 / 40 \mathrm{mmHg}$, pulse rate 100 beats $/ \mathrm{min}$, temperature $36.4^{\circ} \mathrm{C}$, puffy face, myxoedema, dry skin, delayed relaxation of ankle reflexes, normal jugular pulse and poorly localisable apex beat. The investigations on admission revealed serum $\mathrm{Na}^{+} 137 \mathrm{mmol} / 1, \mathrm{~K}^{+} 3.8 \mathrm{mmol} / \mathrm{l}$, triiodothyronine $0.09 \mathrm{nmol} / 1$ (normal range $1.2-2.4 \mathrm{nmol} / \mathrm{l}$ ), thyroxine $1.29 \mathrm{nmol} / 1$ (51$155 \mathrm{nmol} / \mathrm{l})$, thyroid-stimulating hormone $1.4 \mathrm{mU} / 1(0-6 \mathrm{mU} / \mathrm{l})$, luteinising hormone $2.0 \mathrm{IU} / \mathrm{l}$, follicle-stimulating hormone $3.2 \mathrm{IU} / 1$ and oestradiol $70 \mathrm{nmol} / \mathrm{l}$. Serum prolactin and cortisol were less than the $2.5 \mu \mathrm{g} / 1$ and $15 \mathrm{nmol} / \mathrm{l}$, respectively. Computed tomography (CT) of the pituitary fossa demonstrated an empty sella. The cardiac echocardiogram and chest X-ray are shown in figures 1 and 2, respectively.

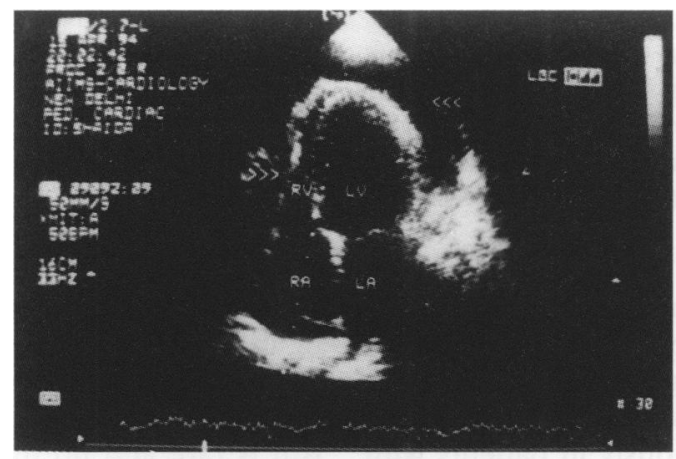

A

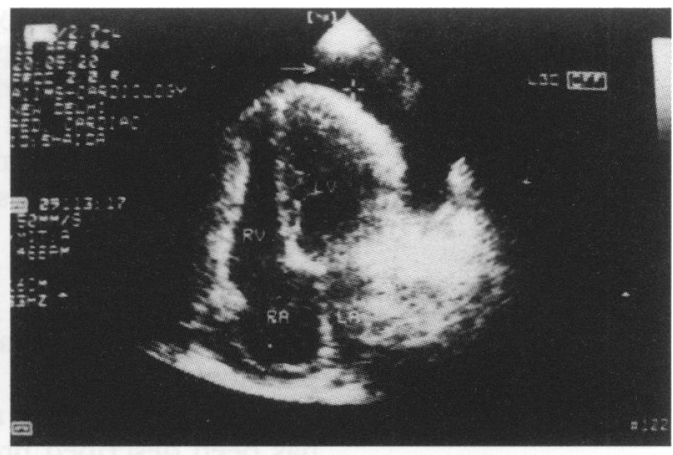

Figure 1 Two-dimensional echocardiography, apical four chamber view in early diastole (A) and early systole (B)

All India Institute of
Medical Sciences, New
Delhi 110029, India
Department of
Endocrinology
R Goswami
N Tandon
P Shah
AC Ammini
Department of
Cardiology
B Singh
Correspondence to
Dr Ammini
Accepted 5 September 1995

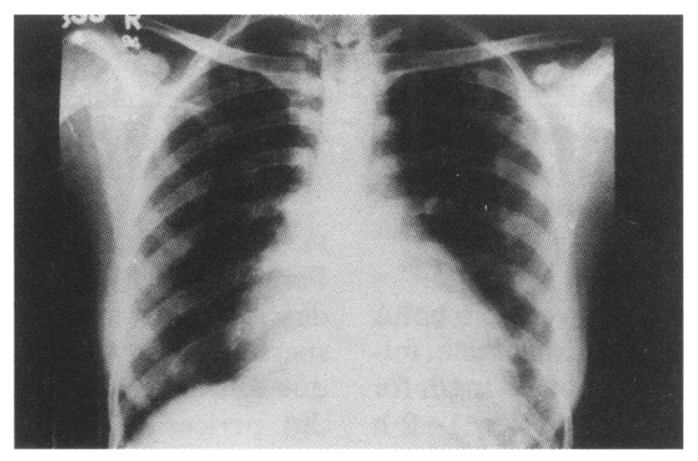

Figure 2 Admission chest X-ray, postero-anterior view

\section{Questions}

1 Describe the findings on chest X-ray and echocardiogram.

2 How do you correlate findings on imaging with the clinical presentation?

3 Which cardinal bedside signs of the condition revealed by imaging are missing and why?

4 How would you manage this case? 


\section{Answers}

\section{QUESTION 1}

Figure 1 is a two-dimensional echo frame showing a large circumferential echo-free zone of $3 \mathrm{~cm}$ with evidence of right ventricular early diastolic collapse. Figure 2 reveals an enlarged cardiac silhouette with no evidence of pulmonary venous congestion. These findings indicate the presence of a large pericardial effusion with tamponade.

\section{QUESTION 2}

There are clinical features of severe hypothyroidism. The history of post-partum haemorrhage, hormone profile and empty sella on pituitary fossa imaging suggest it to be central in origin. The presence of circulatory collapse in this patient appears to be consequent to two pathologies: massive pericardial effusion and adrenocortical insufficiency. The occurrence of a massive pericardial effusion in a patient with secondary hypothyroidism is unusual; such patients usually present early to the physician with multiple complaints resulting from the deficiency of other pituitary hormones. As a consequence, hypothyroidism is never gross. However, in developing countries, patients often report late during the course of their illness, as exemplified by the present case who tolerated the symptoms of hypopituitarism for four years prior to hospitalisation.

\section{QUESTION 3}

In spite of the large pericardial effusion and right ventricular diastolic collapse, pulsus paradoxus and raised jugular venous pressure were absent. Similar absence of clinical signs has been described previously in patients with cardiac tamponade and coexisting volume depletion. ${ }^{1}$ The rise of right atrial pressure is less marked in the presence of hypovolaemia. Thus, cardiac tamponade may be masked when hypovolaemia is present. This type of tamponade is called 'low pressure tamponade'. ${ }^{1}$ In this patient the cardiac tamponade associated with myxoedema was of the low-pressure type, presumably due to the presence of hypovolaemia associated with hypocortisolism.

\section{QUESTION 4}

Acute treatment in this patient involved a bolus of $100 \mathrm{mg}$ hydrocortisone hemisuccinate followed by an infusion at the rate of $10 \mathrm{mg} / \mathrm{h}$ for at least $24 \mathrm{~h}$. A fluid load (1 $1 / \mathrm{h}$ for $1-2 \mathrm{~h}$ followed by $500 \mathrm{ml} / \mathrm{h}$ ) of $5 \%$ dextrose in saline $(154 \mathrm{mmol} / \mathrm{l})$ was given. This volume load is helpful not only in the hypocortisolic state but also in massive pericardial effusion. Volume infusion is the standard therapy in cardiac tamponade and has been shown to delay the appearance of right ventricular collapse and haemodynamic deterioration. ${ }^{1}$ If haemodynamic stabilisation is achieved with glucocorticoid replacement and volume replacement, pericardiocentesis can be deferred and the patient closely monitored on thyroxine replacement. Pericardial effusion resolves completely with thyroxine replacement during a follow-up period of 1-12 months. ${ }^{1}$
Pericardiocentesis may be required in occasional cases of cardiac tamponade associated with myxoedema where the patient is haemodynamically unstable, despite fluid load. It is also indicated in those rare cases where worsening is reported following thyroxine of replacement. $^{2}$ Cyclical oestrogen-progesterone can be continued in the presence of an hypoestrogenic state.

The chest X-ray may continue to show an enlarged cardiac silhouette after the disappearance of pericardial effusion (as confirmed by an echocardiogram) due to underlying myxoed- $\bar{\partial}$ matous and ischaemic heart involvement. ${ }^{1}$

\section{Discussion}

\section{HYPOTHYROIDISM AND PERICARDIAL}

EFFUSION

The cardiovascular abnormalities associated with hypothyroidism are shown in box 1 . Pericardial effusion is the most common of these. ${ }^{1}$

\section{PREVALENCE}

The association of pericardial effusion with hypothyroidism was first reported by Gordon in $1929 .{ }^{3}$ About one and a half decades ago, 윽 the prevalence of pericardial effusion in hypothyroidism was reported to be as high as $30 \% .^{1}$ In contrast, recent reports indicate echocardiographically detectable pericardial $\stackrel{\oplus}{\oplus}$ fluid to be present in only $3-6 \%$ of hypothyr- $\vec{\bullet}$ oid patients. This may be due to the early detection of hypothyroidism nowadays because of increased awareness among physicians, as well as the easy availability of thyroid function tests. Cardiac tamponade due to hypothyroidism is rare and up to now only 21 cases have been described in the literature. ${ }^{4}$

\section{PATHOGENESIS}

The effusive serositis of pericardial effusion in myxoedema has been attributed to increased capillary permeability, slow lymphatic drainage, and enhanced avidity for salt and water. ${ }^{1}$ Risk factors for the cardiac tamponade are shown in box 2 .

\section{FLUID CHARACTERISTICS}

Pericardial fluid in myxoedema has been $\frac{7}{0}$ described as clear, straw-coloured, with a specific gravity of 1012-1025. Occasionally, N due to the breakdown of serum lipoproteins in the pericardial cavity, yellowish scintillating

\begin{tabular}{|l|l|}
\hline Cardiac manifestations of \\
hypothyroidism
\end{tabular}

Box 1 


\begin{tabular}{|l|}
\hline $\begin{array}{l}\text { Risk factors } \\
\text { for cardiac } \\
\text { tamponade }\end{array}$ \\
\hline - infection \\
- spontaneous \\
pericardial \\
haemorrhage due \\
to rupture of \\
fragile capillaries \\
- thyroxine therapy \\
- effect of \\
abdominal \\
paracentesis \\
\hline
\end{tabular}

Box 2 particulate matter is seen when fluid is agitated (gold paint appearance). Cytological examination usually demonstrates a lymphocyte count of $0.5 \times 10^{9} / 1$ with occasional polymorphs. The effusion is characterised by a high protein content with a significantly elevated concentration of alpha- and beta-globulin.

\section{CLINICAL FEATURES}

\section{Aetiology of hypothyroidism}

Pericardial effusion is typically considered to be a manifestation of primary hypothyroidism, although no definite relationship has been established between the aetiology of hypothyroidism and the presence of pericardial effusion. Both adolescents and adults with primary hypothyroidism are susceptible to the association. However, patients with congenital hypothyroidism, especially due to agenesis and dyshormonogenesis, are more likely to exhibit pericardial effusion.

In contrast, the heart is classically described as small in patients with secondary hypothyroidism. The absence of pericardial effusion in these patients could be because the hypothyroidism is usually mild and most patients seek medical attention early in the disease course. However, in developing countries, patients with longstanding untreated secondary hypothyroidism may develop gross features of myxoedema and present with pericardial effusion, as exemplified in the presented case and in an earlier one described by Tahir. ${ }^{5}$ Thus, it appears that the severity of the hypothyroidism rather than its aetiology is the major determinant of the presence or absence of pericardial effusion.

\section{Manifestations}

The amount of fluid in the pericardial effusion associated with hypothyroidism may be as large as 5-61. The slow accumulation allows distension of the pericardium with no significant increase in the pericardial pressure. In addition, demands for cardiac output are low in patients with hypothyroidism. These factors account for the rarity of the symptoms and haemodynamic dysfunction, in spite of massive fluid accumulation. ${ }^{1}$ Occasional patients may be symptomatic and even manifest features compatible with cardiac tamponade. In myxoedematous patients with pericardial effusion, the occurrence of low voltage $Q R S$ and tachycardia may be predictive of cardiac tamponade. Symptomatic patients may present with breathlessness, pedal oedema, cyanosis, syncope and circulatory collapse. Examination reveals the usual signs of pericardial effusion (box 3). ${ }^{1}$ Pulsus paradoxus may be absent in spite of massive fluid accumulation. A rare type of cardiac tamponade described as 'low-pressure tamponade', characterised by the absence of both cervical venous distension and pulsus paradoxus, has been reported in occasional patients with hypothyroidism. ${ }^{1}$ Reduced plasma volume in hypothyroidism may be responsible for the appearance of low pressure tamponade.

\section{Signs of pericardial effusion}

- distension of cervical veins and negative Kussmaul's sign

- impalpable cardiac apex with or without pericardial knock

- Ewart's sign

\section{Box 3}

\section{INVESTIGATIONS}

\section{Chest $X$-ray}

On a chest $\mathrm{X}$-ray, a large pericardial effusion appears as an increased cardiothoracic ratio. An apparent increase in cardiac size may be due to myxoedematous myocardial involvement, ischaemic heart disease, or a combination of these factors. However, the sensitivity of the chest X-ray in detecting pericardial effusion is poor and echocardiographically proven effusion has been reported to occur with a radiologically normal heart. ${ }^{1}$

\section{Electrocardiogram}

Some abnormalities of electrocardiogram are almost always associated with pericardial effusion in a hypothyroid patient. These include flattening or inversion of $\mathrm{T}$-wave and low amplitude of $\mathrm{P}, \mathrm{QRS}$ and T-waves. However, these changes are not specific for pericardial effusion and are also seen in hypothyroidism without pericardial effusion. ${ }^{1}$

\section{Echocardiogram}

This is the most reliable means of detecting pericardial effusion and can detect as little as $20 \mathrm{ml}$ of fluid. Echocardiographic signs of significant pericardial effusion are: large circumferential echo-free space, early diastolic right ventricular collapse and compressed left ventricular cavity. Both diastolic right atrial and ventricular collapse occur during the development of cardiac tamponade. Right ventricular collapse is more predictive of cardiac tamponade. ${ }^{1}$

\section{TREATMENT}

Myxoedematous pericardial effusions tend to regress slowly and ultimately disappear over a period of 1-12 months of thyroxine replacement therapy. ${ }^{1}$ In exceptional cases worsening of pericardial effusion has been described with thyroxine treatment. ${ }^{2}$ This is presumed to be due to the precipitation of cholesterol crystal and development of cholesterol pericarditis.

In cases complicated by symptomatic cardiac tamponade rapid relief is desirable and pericardiocentesis is indicated. ${ }^{1}$ In massive pericardial effusion with preserved arterial pressure, the necessity of pericardiocentesis is questionable.

\section{Conclusion}

Pericardial effusion is the most common cardiac complication of hypothyroidism. Usually pericardial fluid accumulation is asymptomatic in hypothyroid patients. How- 


\begin{tabular}{l} 
Cardiac tamponade and \\
hypothyroidism \\
\hline Prevalence \\
- rare (21 cases reported) \\
\\
Hypothyroidism: aetiology \\
- primary hypothyroidism \\
- rarely secondary hypothyroidism (Sheehan's \\
syndrome) \\
Clinical manifestations \\
- usual manifestations (eg, pulsus paradoxus and \\
raised jugular venous pressure) may be absent \\
(low-pressure tamponade) \\
Treatment \\
- haemodynamically stable: thyroxine \\
replacement \\
- haemodynamically unstable: volume \\
replacement, paracentesis, thyroxine \\
replacement \\
Pericardial fluid characteristics \\
- clear, straw coloured \\
- gpecific gravity $1012-1025$ \\
- lymphocyte count around $0.5 \times 10^{9} / 1$ \\
\hline
\end{tabular}

Box 4

\section{Pericadial effusion and hypothyroidism}

Prevalence

- hypothyroidism of all severity: 3-6\%

- myxoedema: $30 \%$

Hypothyroidism: aetiology

- congenital: common

- primary: common

- secondary: rare

Clinical manifestations

- breathlessness

- pedal oedema

- cyanosis

- syncope

- raised jugular venous pressure $+/-$, negative

Kussmaul's sign

- pericardial knock +/-, Ewart's sign

Diagnosis

- chest X-ray: increased cardiac size

- ECG: nonspecific T-wave changes, low QRS voltage

- echocardiogram: circumferential echo-free zone

Treatment

- thyroxine replacement

Box 5

be of the 'low pressure' type, due to associated hypovolaemia of hypothyroid and hypocortosolic state.

\section{Final diagnosis}

Pericardial effusion in a patient with secondary hypothyroidism.

Keywords: pericardial effusion, secondary hypothyroidism, cardiac tamponade, Sheehan's syndrome

4 Manolis AS, Variable P, Ostrowski RM. Hypothyroid cardiac tamponade. Arch Intern Med 1987; 147: 1167-9.

5 Tahir MZ. Cardiac tamponade in hypothyroidism due to Sheehan's syndrome. F Pakistan Med Assoc 1992; 42: 249 